\title{
Development of a Free-Free Transverse Beam Model Using Lateral Vibrations of Beam Conventional Method during Seismic Activity
}

\author{
M.A. Salim \\ Faculty of Mechanical Engineering, Universiti Teknikal Malaysia Melaka \\ Hang Tuah Jaya, 76100 Durian Tunggal, Melaka, Malaysia \\ E-mail: azli@utem.edu.my \\ A. Noordin \\ Faculty of Electrical Engineering, Universiti Teknikal Malaysia Melaka \\ Hang Tuah Jaya, 76100 Durian Tunggal, Melaka, Malaysia \\ E-mail: aminurrashid@utem.edu.my \\ M.Z. Akop \\ Faculty of Mechanical Engineering, Universiti Teknikal Malaysia Melaka \\ Hang Tuah Jaya, 76100 Durian Tunggal, Melaka, Malaysia \\ E-mail: zaid@utem.edu.my
}

Received: December 16, 2010 Accepted: January 6, 2011 doi:10.5539/jmr.v3n3p77

The research is financed by Faculty of Mechanical Engineering, Universiti Teknikal Malaysia Melaka, Malaysia.

\begin{abstract}
This paper expresses the derivation of Free-Free Transverse Beam Model using lateral vibration of beam conventional method during Seismic Activity. Derivation from three mathematical models gives $\cosh \beta L \cos \beta L=1$. Then by numerical software, the graph of those mathematical models is plotted. From the plots, and using equations, the natural frequencies of those three models are identified at values of $389.5 \mathrm{rad} / \mathrm{s}, 2440.9 \mathrm{rad} / \mathrm{s}$, and $6825 \mathrm{rad} / \mathrm{s}$ for $\omega_{1}, \omega_{2}$ and $\omega_{3}$ respectively.
\end{abstract}

Keywords: Free-free beam, Transverse beam, Lateral, Mathematical model

\section{Introduction}

Vibration is mitigated as the relative motion which takes place between the structure and the mass via the dashpot. This kind of energy dissipation allows the beam to obtain some of the external force in place of the structure for stabilization.

Vibration is defined as a periodic back and forth motion of the particles of an elastic body or medium. It is usually a result of the displacement of a body from an equilibrium condition, followed by the body response to the forces that tend to restore equilibrium (Shirley 1996). It is also considered as the transfer between the kinetic energy and potential energy. It means that the vibration systems have their energies both stored and released. Recently, it has been argued that vibration refers to mechanical oscillations about an equilibrium point.

Two approaches can be taken to help high risk building withstands from the vibrations on its structure. The first involves the design of the structure with the combination of sufficient strength, stiffness and also with an elastic deformation capacity to withstand the vibration from various sources. This can be done by using the combination of structural components such as shear wall, braced frames, moment resisting frames, diaphragms and horizontal trusses to form lateral load resisting system. The soil of the structure is the influencing factor that gives impact on the vibration characteristics and the amount of damage structure sustained. As a result, certain level of deformation and damage occurs (Beards, 2000).

The second approach is using the suitable beam that is designed to absorb vibration energy on the building which its target is to reduce all the high risk structural responses on displacement, velocity and acceleration behaviors. The beam must be designed in a way to produce a good response that can be depended on the structure collapse during the vibration (M.A.Salim, 2006). Many designs of beams can be adopted such as free-free beam, fix-free beam, fix-roller beam and many more. In this study, the free-free beam had been chosen and it was redesigned using a novel approach to give more suitable in usage for high risk buildings during vibration activity (M.A.Salim, 2006), (M.A.Salim, 2009), (Mohd Azli Salim, 2010). The one, two and three dimensional mathematical models were derived and finally the novelty free-free beam is known as the free-free transverse beam. According to this novelty beam, the mode shape for the first, second and third natural frequencies are plotted using numerical software and the result in term of the graphical shape is better than 
before.

\section{Analytical model}

In this study, there are three model were developed to analyze the behavior of the free-free beam when the seismic activity happens. There are:

1. One dimensional mathematical model

2. Two dimensional mathematical model

3. Three dimensional mathematical model

All of these three mathematical models were derived and are shown in equation 1 till equation 23 below.

$<$ Figure $1>$

The initial boundary condition of this free-free beam is $x=0$ when the distance is defined at the left point and $x=L$ when the boundary is taken from the right point.

The one dimensional mathematical model can be derived by using the equation 1 below:

$$
\frac{d y}{d x}=0
$$

Two dimensional mathematical models can be stated derived by using equation 2 .

$$
\frac{d^{2} y}{d^{2} x}=0
$$

And three dimensional mathematical models can be derived by using equation 3 .

$$
\frac{d^{3} y}{d^{3} x}=0
$$

By using the equation of wave propagation in beam, the initial equation of free-free beam can be stated in equation 4 .

$$
y=A \sinh \beta x+B \cosh \beta x+C \sin \beta x+D \cos \beta x
$$

Then, equation 4 was applied to the equation 1,2 and 3 and the new equations are shown in equation 5, 6 and 7.

$$
\begin{aligned}
\frac{d y}{d x} & =\beta[A \cosh \beta x+B \sinh \beta x+C \cos \beta x-D \sin \beta x] \\
\frac{d^{2} y}{d x^{2}} & =\beta^{2}[A \cosh \beta x+B \sinh \beta x-C \cos \beta x-D \sin \beta x] \\
\frac{d^{3} y}{d x^{3}} & =\beta^{3}[A \cosh \beta x+B \sinh \beta x-C \cos \beta x+D \sin \beta x]
\end{aligned}
$$

The initial boundary condition of $x=0$ was set and the boundary condition was applied into the equation 6 . The free-free beam equation then was changed to the novel equation of free-free transverse beam and is shown in the equation below.

$$
\begin{gathered}
\frac{d^{2} y}{d x^{2}}=\beta^{2}[A \cosh \beta(0)+B \sinh \beta(0)-C \cos \beta x-D \sin \beta(0)] \\
\frac{d^{2} y}{d x^{2}}=\beta^{2}[A(0) x+B(1)-C(0)-D(0)]
\end{gathered}
$$

Then, an assumption was made that equation 8 is equal to zero. The new equation can be expressed as equation 9 below.

$$
\begin{gathered}
\beta^{2}[A(0)+B(1)-C(0)-D(0)]=0 \\
B-D=0 \\
B=D
\end{gathered}
$$


The initial boundary condition is used again and for this time the boundary condition is applied into equation 7 .

$$
\begin{gathered}
\frac{d^{3} y}{d x^{3}}=\beta^{3}[A \cosh \beta x+B \sinh \beta x-C \cos \beta x+D \sin \beta x] \\
\frac{d^{3} y}{d x^{3}}=\beta^{3}[A(1)+B(0)-C(1)+D(0)]
\end{gathered}
$$

The same assumption is also made for equation 10. The new equation is:

$$
\begin{gathered}
\beta^{3}[A(1)+B(0)-C(1)+D(0)]=0 \\
A-C=0 \\
A=C
\end{gathered}
$$

The boundary condition is set at $x=L$. This boundary condition is applied to second derivative (equation 6) of free-free transverse beam. The unknown constants of $\mathrm{C}$ and $\mathrm{D}$ are changed to $\mathrm{A}$ and $\mathrm{B}$, respectively. The reason is to make the equation more easily to be solved accordingly. The new equation is expressed as below.

$$
\frac{d^{2} y}{d x^{2}}=\beta^{3}[A \sinh \beta L+B \cosh \beta L-A \sin \beta L-B \cos \beta L]
$$

Then, assumption is made to equation 12 , to be equal to zero.

$$
\begin{gathered}
\beta^{3}[A \sinh \beta L+B \cosh \beta L-A \sin \beta L-B \cos \beta L]=0 \\
A \sinh \beta L+B \cosh \beta L-A \sin \beta L-B \cos \beta L=0 \\
A(\sinh \beta L-\sin \beta L)+B(\cosh \beta L-\cos \beta L)=0
\end{gathered}
$$

The same boundary condition is then applied into the third derivative (equation 7). The unknown also is changed as the same as equation 12 .

$$
\frac{d^{3} y^{2}}{d x}=\beta^{3}[A \cosh \beta L+B \sinh \beta L-A \cos \beta L+B \sin \beta L]
$$

An assumption is made to equation 14 to be equal to zero. The new equation can be expressed by the equation below.

$$
\begin{gathered}
\beta^{3}[A \cosh \beta L+B \sinh \beta L-A \cos \beta L+B \sin \beta L]=0 \\
A \cosh \beta L+B \sinh \beta L-A \cos \beta L+B \sin \beta L=0 \\
A(\cosh \beta L-\cos \beta L)+B(\sinh \beta L+\sin \beta L)=0
\end{gathered}
$$

The equations 13 and 15 were rearranged into matrix form. The purpose of this matrix form is to identify the governing equation of free-free transverse beam. Besides that, matrix form is used to represent the equations into more simple form. The matrix form of the equations can be represented as below.

Equation 10 and 12 in matrix form is:

$$
\left[\begin{array}{cc}
(\sinh \beta L-\sin \beta L) & (\cosh \beta L-\cos \beta L) \\
(\cosh \beta L-\cos \beta L) & (\sinh \beta L+\sin \beta L)
\end{array}\right]\left[\begin{array}{l}
A \\
B
\end{array}\right]=\left[\begin{array}{l}
0 \\
0
\end{array}\right]
$$

Then, the equation 16 is rearranged to solve the governing equation of free-free transverse beam.

$$
\begin{gathered}
(\sinh \beta L-\sin \beta L)(\sinh \beta L+\sin \beta L)-(\cosh \beta L-\cos \beta L)(\cosh \beta L-\cos \beta L)=0 \\
{\left[\left(\sinh ^{2} \beta L\right)+(\sinh \beta L \sin \beta L)+(-\sin \beta L \sinh \beta L)+\left(-\sin ^{2} \beta L\right)\right]} \\
-\left[\left(\cosh ^{2} \beta L\right)+(-\cosh \beta L \cos \beta L)+(-\cos \beta L \cosh \beta L)+\left(\cos ^{2} \beta L\right)\right]=0
\end{gathered}
$$




$$
\begin{gathered}
\sinh ^{2} \beta L+\sinh \beta L \sin \beta L-\sin \beta L \sinh \beta L-\sin ^{2} \beta L \\
-\cosh ^{2} \beta+\cosh \beta L \cos \beta L+\cos \beta L \cosh \beta L-\cos ^{2} \beta L=0 \\
\sinh ^{2} \beta L-\sin ^{2} \beta L-\cosh { }^{2} \beta L+\cosh \beta L \cos \beta L+\cos \beta L \cosh \beta L-\cos ^{2} \beta L=0 \\
\sinh ^{2} \beta L-\sin ^{2} \beta-\cosh { }^{2} \beta L+2 \cosh \beta L \cos \beta L-\cos ^{2} \beta L=0
\end{gathered}
$$

The trigonometric identity is applied in Equation 20 in order to solve it.

$$
\begin{gathered}
\left(\frac{-1+\cosh 2 \beta L}{2}\right)-\left(\frac{1-\cos 2 \beta L}{2}\right)-\left(\frac{(1+\cosh 2 \beta L)}{2}\right)+2 \cosh \beta L \cos \beta L-\left(\frac{1+\cos 2 \beta L}{2}\right)=0 \\
-\frac{1}{2}+\frac{\cosh 2 \beta L}{2}-\frac{1}{2}+\frac{\cos 2 \beta L}{2}-\frac{1}{2}-\frac{\cosh 2 \beta L}{2}+2 \cosh \beta L \cos \beta L-\frac{1}{2}-\frac{\cos 2 \beta L}{2}=0 \\
-2+2 \cosh \beta L \cos \beta L=0
\end{gathered}
$$

The new equation of free-free transverse beam is shown in equation 23:

$$
\begin{gathered}
2 \cosh \beta L \cos \beta L=2 \\
\cosh \beta L \cos \beta L=1
\end{gathered}
$$

\section{Results and discussion}

Based on the one, two and three dimensional mathematical models, there are three graphs can be plotted and they are:

1. First mode shape

2. Second mode shape

3. Three mode shape

The natural frequency equation is shown in equation 24 .

$$
\omega_{n}=\beta^{2} \sqrt{\frac{E I}{\rho A}}
$$

In this study, aluminum material was used because aluminum is one part of metal material. Besides, the density of this material is low but the Young Modulus E is high. According to this reason, aluminum has been chosen in this study. From the lateral vibrations of beams, the mode shape can be identified. For the first mode shape, the value of $\beta L=1.875104$ and $\beta=1.8751042$. Therefore, the first natural frequency is:

$$
\omega_{1}=0.879 \times 443.125=389.5 \mathrm{rad} / \mathrm{s}
$$

For second mode shape, the value of $\beta L=4.694091$ and $\beta=4.694091 / 2$. The second natural frequency is:

$$
\omega_{2}=5.5086 \times 443.125=2440.9 \mathrm{rad} / \mathrm{s}
$$

Lastly for third mode shape, the value of $\beta L=7.854757$ and $\beta=7.8547572$. The third natural frequency is:

$$
\omega_{3}=15.4243 \times 443.125=6825 \mathrm{rad} / \mathrm{s}
$$

All the first, second and third mode shape graph is shown in Figure 2, Figure 3 and Figure 4, respectively.

$<$ Figure 2-4 > 


\section{Conclusion}

In this study, the one, two and three dimensional mathematical model from free-free beam are derived by using the lateral vibration of beams method. Based on this method, the free-free beam equation has changed to free-free transverse beam equation and the final equation is $\cosh \beta L \cos \beta L=1$. Besides that, according to this lateral vibration of beams method, the analytical analysis of first, second and third natural frequencies of the free-free beam can be identified. By using the numerical software, the graph of these three mode shapes has been shown in Figure 2. (a), (b) and (c) for the first, second and third mode shapes, respectively.

\section{Acknowledgement}

The author would like to thank to the Faculty of Mechanical Engineering, Universiti Teknikal Malaysia Melaka, Malaysia.

\section{References}

C.F. Beards. (2000). JStructural vibrations: Analysis and damping, New York, Arnold Ltd. pp 3.

J.D. Shirley. (1996). Acceleration feedback control strategies for active and semi active control system: Modeling, algorithm development and experimental verification, Civil Engineering and Geological Sciences Notre Dame. Indiana.

M.A.Salim. (2006). Design a control system to dampen the vibration in a building like structure, Theses. Kolej Universiti Teknikal Kebangsaan Malaysia.

M.A.Salim. (2009). Analysis of absorption the level of vibration energy in a building structure using PID controller., ICORAFSS 2009. pp 99-105.

Mohd Azli Salim, Mohd Khairi Mohamed Nor, \& Aminurrashid Noordin. (2010). Vibration on building: A stability analysis using PID controller, Journal of Materials Science and Engineering. USA. Volume 4. No 5. pp 71-79.

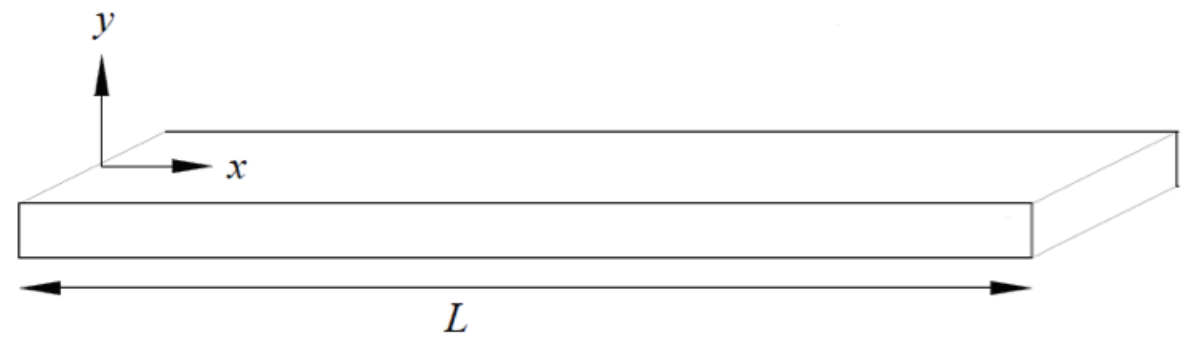

Figure 1. Free-free beam in steady state condition

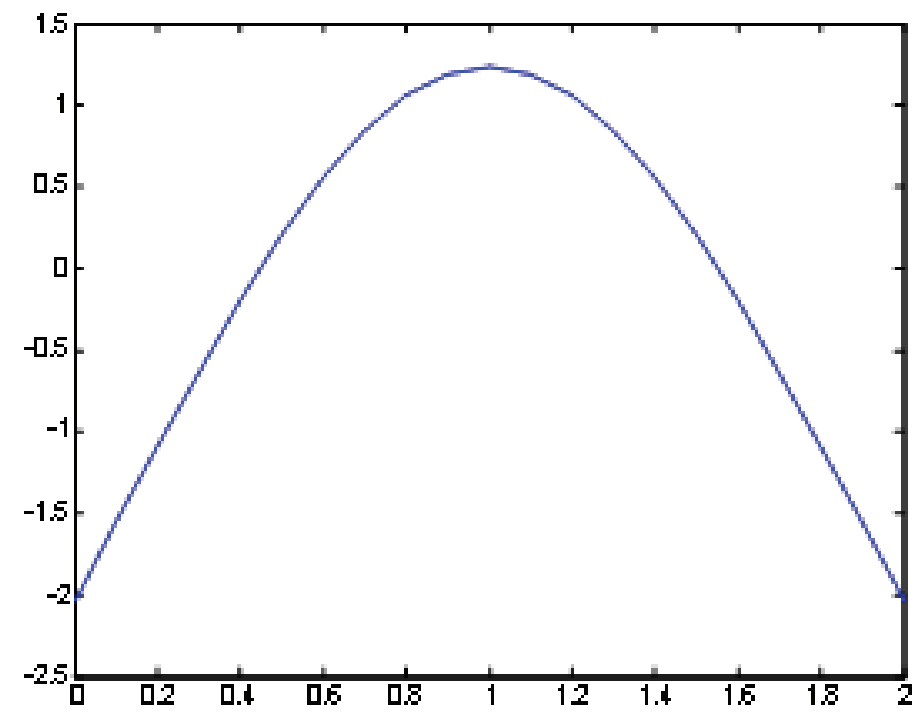

Figure 2. $1^{\text {st }}$ mode shape 


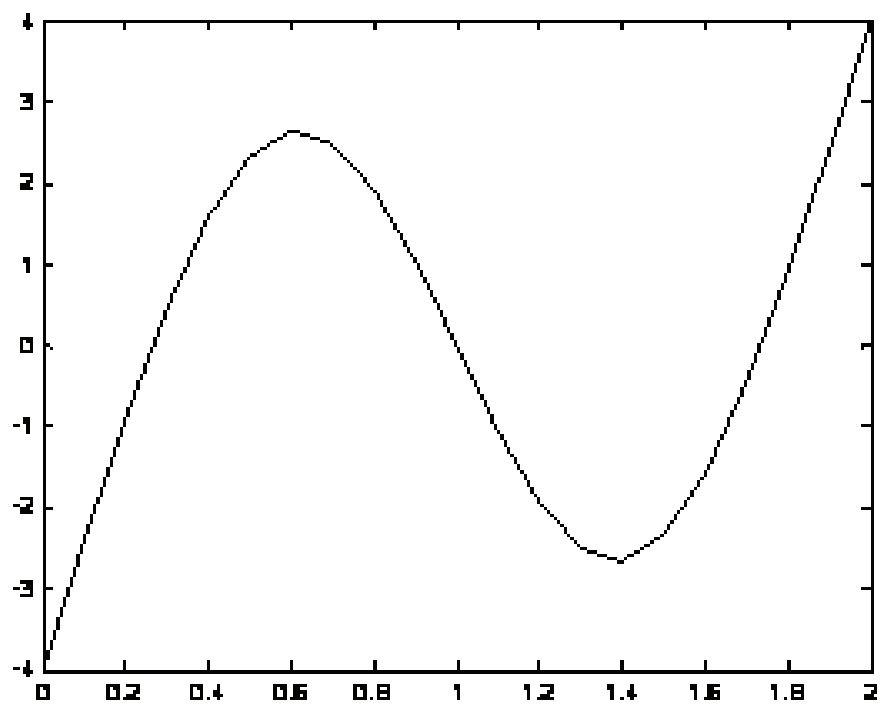

Figure $3.2^{\text {nd }}$ mode shape

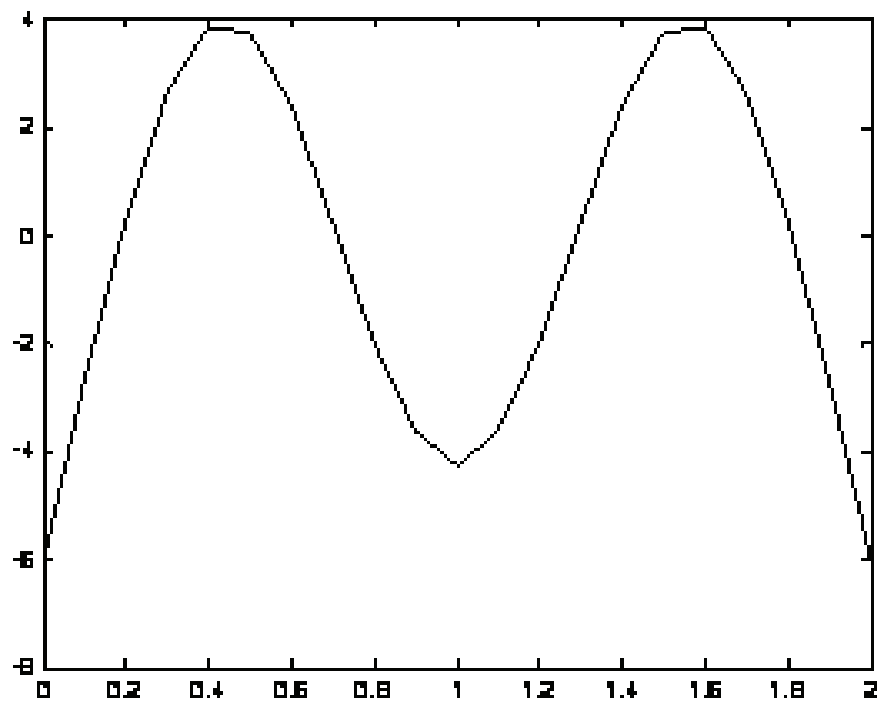

Figure 4. $3^{r d}$ mode shape 